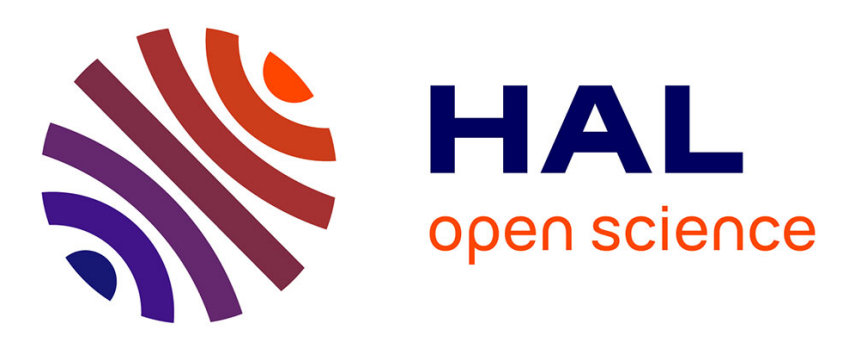

\title{
La prise en compte des émotions : une solution pour les logiciels éducatifs pour enfants déficients intellectuels
} Gabriel Michel, Magali Jobert

\section{To cite this version:}

Gabriel Michel, Magali Jobert. La prise en compte des émotions: une solution pour les logiciels éducatifs pour enfants déficients intellectuels. STICEF (Sciences et Technologies de l'Information et de la Communication pour l'Éducation et la Formation), 2008, 15, 7 p. hal-00696409

\section{HAL Id: hal-00696409 \\ https://hal.science/hal-00696409}

Submitted on 11 May 2012

HAL is a multi-disciplinary open access archive for the deposit and dissemination of scientific research documents, whether they are published or not. The documents may come from teaching and research institutions in France or abroad, or from public or private research centers.
L'archive ouverte pluridisciplinaire HAL, est destinée au dépôt et à la diffusion de documents scientifiques de niveau recherche, publiés ou non, émanant des établissements d'enseignement et de recherche français ou étrangers, des laboratoires publics ou privés. 


\title{
La prise en compte des émotions : une solution pour les logiciels éducatifs pour enfants déficients intellectuels
}

\author{
Gabriel MICHEL*, Magali JOBERT** \\ [*ETIC, Université Paul Verlaine, Metz ; **ErgoClic, Metz $]$
}

- RÉSUMÉ : Les enfants déficients cognitifs sont oubliés par les technologies éducatives. Nous montrons d'abord qu'il existe peu de logiciels qui leur sont adaptés. Notre travail avec ces enfants, les éducateurs et les parents a abouti à la conception d'un didacticiel spécifique plus compatible que les logiciels actuels et a permis de construire un certain nombre de recommandations ergonomiques. Mais pour aller plus loin, nous avons fait le pari de l'introduction des émotions dans nos interfaces. Nous avons reconstruit un univers virtuel qu'on ne trouve pas dans les didacticiels actuels, souvent neutres et lisses : il s'agit d'un univers de contes qui relate des histoires crues qui semblent réelles et qui sont souvent plus proches de la réalité de ces enfants. Les premières évaluations de ce didacticiel sont prometteuses.

MOTS CLÉS : IHM, émotions, apprentissage, didacticiel, enfants déficients intellectuels, déficients cognitifs, recommandations.

aBSTRACT : There exists very little educational software for intellectually handicapped children. The assessment that we carried out of software currently in use proves that there exists little software adapted for these children. Work accomplished with teachers and children led to the design of a specific educational software application, which is more compatible than the current software available. This work also made it possible to build a number of ergonomic guidelines. But to go further, we introduced emotions into our interfaces. We rebuilt a virtual universe, which one does not find in the current educational software : it concerns the universe of fairytales, which tell stories that seem real and which are often closer to the reality of these children. The first evaluations of this educational software application are promising.

KEYWORDS : HCI, émotions, learning, didacticiel, intellectually handicapped, cognitive handicapped, guidelines.

- 1 . Introduction

- $\underline{2 .}$ Ergonomie des didacticiels actuels et premières recommandations

- 3 . Emotion et cognition

- 4 . Nos hypothèses

○ $\underline{\text {. Didacticiel développé }}$

○ $\underline{\text { 6. Evaluation }}$

$\circ \underline{7 .}$ Discussion et conclusion

- $\underline{\text { 8. Bibliographie }}$

\section{I'troductio'}

De plus en plus les enfants apprennent en jouant sur des ordinateurs, à l'école ou à la maison. Les didacticiels qui leur sont consacrés sont de plus en plus nombreux et on voit apparaître un certain nombre de recommandations pour leur conception. Mais pour les enfants déficients cognitifs, ces recommandations ergonomiques en sont à leurs débuts. Signalons quelques groupes de recherche travaillant sur ce sujet comme le projet WAI-NOT en Belgique (WAI-NOT, 2007) et le NCDDR "Centre 
National pour la Diffusion de la Recherche sur les Déficiences" aux Etats-Unis (NCDDR, 2007). L'apport des logiciels éducatifs pour les déficients cognitifs est évidente quand on travaille sur le terrain (Sperandio et al., 1999) et il est dommage qu'ils n'en profitent pas d'avantage.

Pour réaliser ce travail, nous avons adopté une démarche centrée-utilisateurs courante en ergonomie. Notre recherche a tout naturellement commencé par une étude préalable sur les didacticiels utilisés par ces enfants (en y incluant les logiciels disponibles sur Internet) puis nous avons établi une première liste de recommandations ergonomiques pour la conception et l'évaluation de ce type de logiciels.

A partir de ces recommandations, nous avons construit un logiciel qui a été créé en collaboration avec les éducateurs et les enfants des différents établissements. Malgré les résultats encourageants de ce logiciel

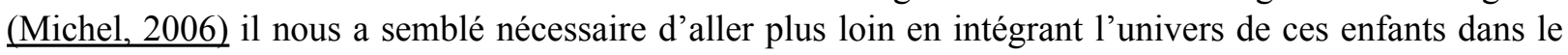
didacticiel. D'où nos hypothèses que nous présentons ainsi que la nouvelle version du logiciel et nos premiers résultats.

\section{Ergo 'omie des didacticiels actuels et premières recomma 'datio's}

\subsection{Les enfants déficients cognitifs}

Pour mieux connaître ces enfants, nous avons, depuis 4 ans, travaillé avec une quinzaine d'éducateurs, une dizaine de parents et aussi observé et discuté avec des élèves de différentes classes. Ces connaissances ont été complétées par une revue de la littérature. Voici quelques concepts marquants que nous avons découverts et qui nous ont guidés lors de la conception des didacticiels (pour plus de détails se référer à (Michel, 2004), (Gascon et al., 2006) :

- Il existe chez les enfants déficients, comme une "peur de l'apprentissage" ou "une interdiction de penser". Ils ont besoin d'être sécurisés, motivés par rapport à leurs expériences d'échecs, de reprendre confiance en eux ;

- En leur donnant le droit à l'erreur sans conséquences, sans jugement, sans sanction d'aucune sorte, ni humiliation, en leur donnant la possibilité d'évoluer à leur propre rythme, aussi lent soit-il, l'ordinateur renvoie aux enfants une image positive d'eux-mêmes ;

- Ils ont très peu accès à la lecture, ne connaissent pas bien "l'extérieur" et en ont peur car cet extérieur est vu comme un ennemi ;

- L'ordinateur leur redonne la certitude de leur propre pensée et de la possibilité d'aboutir à un résultat fini propre et "présentable" même lorsque l'on écrit mal ou que l'on se trompe souvent.

On trouve dans les écoles françaises contactées de très nombreux logiciels "grand public" pour enfants normaux, utilisés "par défaut" pour les enfants déficients et quelques didacticiels spécifiquement conçus pour ces enfants. Ces derniers sont en général peu professionnels car souvent développés par les éducateurs avec peu de moyens, voire bénévolement et tous comportent de nombreuses faiblesses (Michel, 2006). Tous les logiciels utilisés dans les écoles où nous avons mené cette recherche ne sont donc que peu utilisables et la médiation de l'éducateur est souvent nécessaire : on est loin de cette autonomie qu'ils devraient offrir.

\subsection{Premières recommandations}

Il n'existe pas, à notre connaissance, de travaux touchant au sujet qui nous intéresse. Citons deux études très générales concernant la conception de didacticiels telles que celle de (Hû et Trigano, 1998) ou encore celle de (Mataigne et al. 1995) qui a conçu un guide d'évaluation pour logiciels éducatifs pour le Ministère de l'Education Nationale du Québec. Par contre il existe quelques recommandations pour des sites web accessibles. Ces recommandations sont issues du site du WAI (WAI, 2007) mais se différencient peu des recommandations pour tous publics et ne concernent que les déficients cognitifs ayant des notions de lecture.

C'est la raison pour laquelle nous avons choisi de créer une première version d'un logiciel construit en collaboration avec les éducateurs et enfants et ayant intégré les prémisses de recommandations issues de 
nos interviews, enquêtes et études bibliographiques. Il fallait tenir compte des problèmes essentiels de ces enfants tels que le manque de concentration, des problèmes d'attention, une image négative d'eux-mêmes et des problèmes de mémoire. Voici quelques exemples de recommandations (pour des recommandations plus complètes se référer à (Michel et al., 2006) ) :

- Créer un personnage, bienveillant et complice, avec lequel l'enfant puisse s'identifier et interagir ;

- Permettre à l'enfant de choisir ce compagnon de jeu (plutôt que d'apprentissage), qui l'appellera également par son prénom, avec lequel il fera les différents jeux proposés ;

- Utiliser un personnage qui sera "l'assistant vocal" qui énoncera la consigne de l'exercice. L'enfant devra cliquer sur ce personnage pour réécouter la consigne ;

- Prendre en compte le problème de l'âge réel des enfants.

Malgré des résultats des tests utilisateurs convaincants (comparaison sur des séquences d'apprentissage analogues de notre logiciel avec celles des logiciels utilisés dans les écoles), il nous a paru évident que les facteurs classiques de l'utilisabilité (efficacité, efficience, satisfaction et compatibilité) étaient trop limités. C'est la raison pour laquelle nous nous sommes intéressés aux émotions.

\section{Emotio ' et cog 'itio'}

"Aesthetics matter : attractive things work better" (Norman, 2002). Cette affirmation de Norman prouve que l'on pourra augmenter l'utilisabilité des produits par l'esthétique. Mais il n'y a pas que l'esthétique qui peut augmenter l'utilisabilité d'un produit. Depuis quelques années l'intérêt des émotions dans la conception et l'évaluation des interfaces ne cesse de grandir, et beaucoup de groupes de recherches traitant de ce sujet apparaissent. En psychologie ergonomique et en psychologie cognitive on s'intéresse de plus en plus aux liens entre cognition et émotions ou affects : on reconnaît que les émotions ont des effets sur la cognition et la cognition a des effets sur les émotions (Tricot et al., 2003), (Kirouac, 2004), (Blanc, 2006). Etudier les émotions c'est s'intéresser aussi aux affects, au stress, à la motivation. On sait aussi que les émotions ont des effets sur la mémoire ou sur l'attention (Blanc, 2006), (Kirouac, 2004). L'étude des émotions dans l'activité devient également un champ de recherche de l'ergonomie en général (Grosjean et al., 2003).

Il est clair que ces différentes études s'appliquent en particulier aux interfaces informatiques (et aux apprentissages) en essayant de répondre à la question des émotions portées par une interface, des affects qu'elle suscite et des conséquences sur le fonctionnement cognitif des utilisateurs. Certains chercheurs, adeptes de la psychologie positive, avancent que les IHMs et l'ingénierie de l'utilisabilité ont besoin d'un changement de direction (Hassenzahl, 2003): les futures IHMs doivent évoluer dans le sens d'un évitement des aspects négatifs de l'interaction avec les produits (erreurs, stress, anxiété) vers une conception attrayante de l'expérience utilisateur.

\section{Nos hypothèses}

\subsection{Utilisation de contes violents}

Les causes de la déficience cognitive dans la littérature sont pour l'essentiel héréditaires ou accidentelles : mais notre travail sur le terrain avec les éducateurs a mis en évidence une autre hypothèse. En effet, pour un certain nombre d'enseignants que nous avons rencontré, les déficiences cognitives de la plupart des enfants dont ils ont la charge proviennent de leur environnement familial et social. Pour eux la déficience cognitive était dans la majorité des cas liée à la déficience affective et à la violence.

Nous avons fait l'hypothèse que les personnages des didacticiels proposés à ces enfants (mignons, charmants, aimables,...) sont trop éloignés du monde des enfants. Pour cette raison nous avons essayé de reconstruire un espace virtuel de contes qui relatent des histoires crues qui semblent réelles. Dans ces contes les enfants peuvent plus facilement se reconnaître avec l'envie de s'identifier à des personnages victimes ou bourreaux. C'est la raison pour laquelle nous avons choisi l'univers de Peter Pan (créé par James Matthew Barrie).

Nous avons pensé que la réduction des troubles psychologiques occasionnés par l'environnement social des enfants pourrait être détournée temporairement à travers l'interface du logiciel. Ainsi, le rapport dominant/dominé ou enseignant/enseigné dans ce cas peut être inversé ce qui pourrait réduire la frustration due à l'échec, l'angoisse due à des conflits internes perpétuels difficilement surmontables et 
permettre un meilleur contrôle de l'impulsivité.

\subsection{Principes essentiels d'un tel didacticiel}

Pour captiver l'attention de l'enfant, en dehors de la création d'un univers assez agressif, nous avons essayé d'intégrer un certain nombre de dimensions émotionnelles dans le didacticiel : le plaisir (les enfants ont l'impression de jouer et non d'apprendre), la réduction de la peur de l'échec et la joie (en félicitant l'enfant dès qu'il réussit et en lui donnant des récompenses à la fin de chaque exercice réussi).

\section{Didacticiel développé}

Pour tester nos hypothèses, dans le cadre de notre didacticiel que nous avons appelé "Clique et Gagne", nous avons développé deux séquences d'apprentissage correspondant à deux exercices du logiciel Adibou (un des didacticiels les plus utilisés dans les CLIS - Classes d'Intégration Scolaire). Il s'agit d'un exercice de français (reconnaître les lettres dans les mots) et un autre de mathématiques (compter) immergés dans l'univers virtuel des contes.

\subsection{Le rôle de l'enfant et l'importance de Clochette}

Le rôle que nous essayons de donner à l'enfant est un rôle de responsabilité. C'est lui qui va aider les personnages présents dans le jeu à s'en sortir, à se libérer, à réussir. Ce sont les décisions (implicitement les apprentissages) de l'enfant qui le permettront. Clochette n'a qu' un rôle d'assistante, d'amuseuse, d'amie. La durée des différentes situations proposées (ou apprentissages de base) sont assez courtes (une trentaine de secondes) et si l'enfant échoue deux fois de suite on lui donne la réponse en lui disant que la prochaine fois il réussira.

Clochette est un personnage jovial, avec une voix chaleureuse, dépourvu d'agressivité : elle est le compagnon de l'enfant. Elle se présente comme étant petite ("Je ne suis pas plus grande que ta main"), et montre régulièrement qu'elle a besoin de l'enfant ( $«$ Je suis toujours très contente que tu viennes me voir et m'aide à passer mes épreuves"). En même temps Clochette conseille l'enfant, lui pose des questions, le guide : en particulier, en terme d'ergonomie, elle complète le guidage de l'interface graphique (qui se veut très simple). Elle évite à l'enfant d'être perdu en étant constamment présente, et lui rappelle ce qu'il faut faire, varie ses interventions afin de paraître plus vivante (par exemple quand l'enfant réussit, selon le cas, elle lui dit "Excellent, c'est génial" ou "C'est très bien", "Oui c'est exact, félicitations",.....). Elle est d'humeur égale, encourage toujours, le récompense même quand il échoue. Elle fait tout pour devenir complice de l'enfant.

\subsection{Illustration}

Dans l'exercice de français de Clic et Gagne, Clochette dit à l'enfant "Quand je parle, Peter mélange les sons : j'ai vraiment besoin de toi pour lui parler". Clochette propose ainsi un mot, symbolisé par une image, et l'enfant doit dire si Peter Pan entend tel ou tel son. L'écran ci-dessous correspond au dialogue : "Quand tu entends le son i dans le mot souris alors dis-le à Peter en cliquant sur ici". Dans cet exemple on demande à Peter s'il entend le son "a" ou le son "i" dans "souris". 


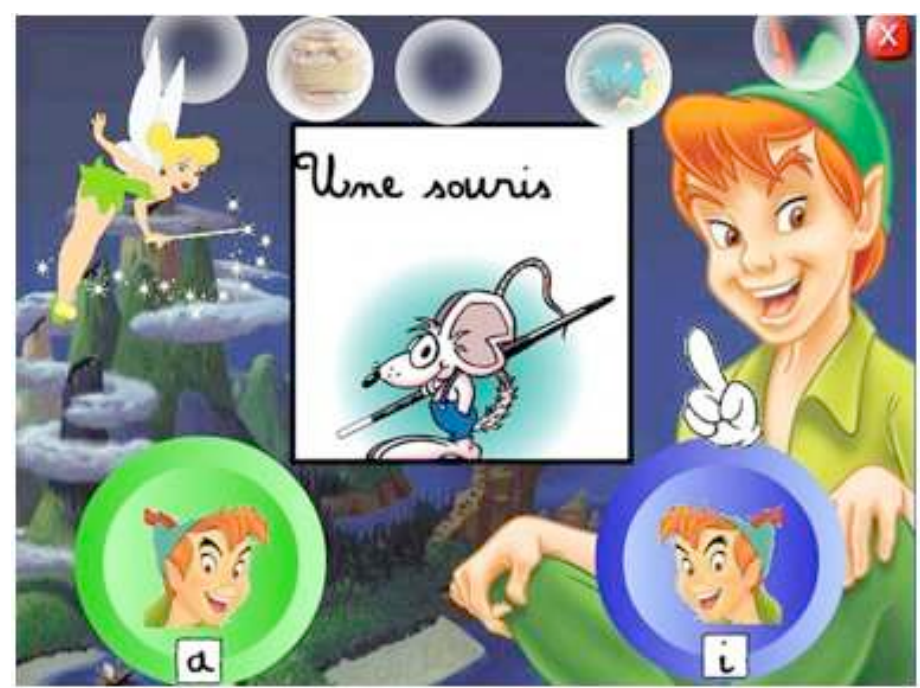

Figure 1・ Exercice de Français

Pour l'exercice de mathématiques (Figure 2 ci-dessous), les enfants perdus, ainsi que Wendy, Jean et Michel ont été kidnappés par le Capitaine Crochet. Ils ont été attachés au mat du bateau des pirates.

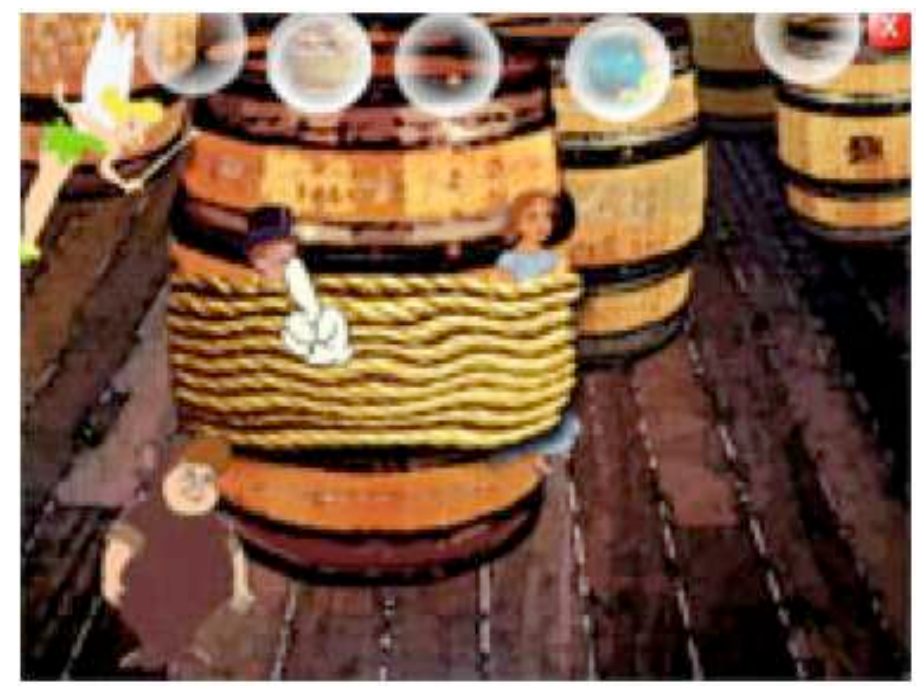

Figure 2 • Exercice de Mathématiques

Clochette demande donc à l'enfant de les libérer (les sortir du panier en pointant avec le doigt) pendant que Peter Pan se bat avec Crochet. Une fois tous les enfants délivrés, l'enfant doit les compter (cf. figure 2), et s'il réussit, les enfants sont définitivement libérés. Dans cet exemple ils sont 6.

\section{Evaluation}

Pour valider nos hypothèses, nous avons mené des tests utilisateurs ayant pour but de comparer notre logiciel et "Adibou 4/5 ans (Ad)". La comparaison entre notre didacticiel "Clique et Gagne (ClG)" et "Adibou 4/5 ans (Ad)" a porté sur deux séquences ayant exactement les mêmes buts et les mêmes tâches : l'une en français l'autre en mathématiques. Nous avons mené des tests utilisateurs avec deux groupes d'enfants de CLIS (Classes d'Intégration Scolaire) : le premier groupe comportait 16 enfants (qui ont testé "Clic et Gagne") et le second 19 enfants (qui ont testé "Adibou") : la moyenne d'âge était de 9 ans dans chacun des 2 groupes. Les enfants ont été observés lors de leurs manipulations à l'aide de grille de codification des comportements : nous avons également rempli avec eux un questionnaire de satisfaction des 2 logiciels. Les résultats sont éloquents. Par exemple, les enfants comprennent la consigne en mathématiques (94\% avec $\mathrm{ClG}$ et $34 \%$ pour Ad). L'enfant aime l'histoire de $\mathrm{ClG}$ dans $62,5 \%$ des cas et $16 \%$ dans le cas d'Ad. Pour Clic et Gagne (par rapport à Adibou), la navigation est beaucoup plus facile, les exercices sont plus souvent réussis, l'enfant est plus autonome (moins de demande d'aide) et le 
maintien de l'attention meilleur. Pour Adibou, $36 \%$ des enfants ont, à un moment donné, manifesté de l'énervement (respectivement $9 \%$ pour $\mathrm{ClG}$ ).

\section{Discussion et conclusion}

Cette recherche nous a permis de souligner la mauvaise ergonomie des didacticiels utilisés actuellement dans l'enseignement dédié aux enfants déficients cognitifs : la difficulté à utiliser ces logiciels rappelant une fois de plus à ces enfants leur différence. Le travail pendant toute l'étude avec ces enfants et leurs éducateurs a abouti à l'élaboration d'un ensemble de recommandations ergonomiques et au développement de différentes versions d'un didacticiel destiné à être utilisé dans un cadre scolaire ou personnel. Même si le respect de ces critères a permis un réelle amélioration de l'intérêt des enfants et de leur l'autonomie, ils nous semblaient encore limités. La prise en compte des émotions dans notre didacticiel par l'intermédiaire de l'univers des contes a permis de franchir encore un pallier dans l'utilisabilité de notre didacticiel : c'est ce que montrent nos dernières évaluations. Pour Clic et Gagne, beaucoup d'enfants sourient d'avantage pendant le jeu et se réjouissent de voir la vidéo à la fin du jeu en guise de récompense. Ils sont très heureux que la fée clochette les reconnaisse, les félicite, les appelle par leurs prénoms. Le monde de Peter Pan a été beaucoup apprécié, car il a le pouvoir de voler, il est libre.

Nous avons ainsi pu confirmer un certain nombre de nos hypothèses. Par exemple que poser des questions aux enfants indirectement leur évitait de risquer de se trouver en échec une fois de plus. Au lieu de leur demander "Trouve la réponse", il est préférable de lui dire "Aide le compagnon de jeu à trouver la réponse" ou "Aide Peter Pan à capturer les pirates". Les enfants se sentent ainsi utiles et développent de la sympathie pour les personnages qu'ils soutiennent et se sentent plus impliqués dans la réussite de l'exercice. Il semble donc que, pour ce type d'enfants, l'importance des émotions dans les apprentissages soit un facteur essentiel et incontournable. Dans une prochaine étape, nous espérons pouvoir améliorer encore notre didacticiel, ajouter d'autres séquences d'apprentissages, d'autres facteurs émotionnels qui permettront par exemple à l'enfant de choisir un compagnon et nous envisageons de faire des tests utilisateurs en laboratoire, ceci afin d'affiner et de compléter nos recommandations et de les mettre à la disposition des concepteurs. Ces concepts nouveaux dans les didacticiels ont été récompensés dans plusieurs concours dont le concours national de création d'entreprises de technologie innovantes du ministère de la recherche en 2007.

\section{Bibliographie}

BLANC N. (2006). Emotion et cognition : Quand l'émotion parle à la cognition, Edition In Press.

GASCON H., BOISVERT D., HAELEWYCK M.C., POULLIN J.R., DETRAUX J.J. (2006). Déficience intellectuelle : savoirs et perspectives d'action, Presses Inter Universitaires, Québec.

GROSJEAN V., RAUFASTE E., GIBOIN A. (Eds.), (2003). Émotions, cognitions et performance : investigations en psychologie ergonomique. Le Travail Humain, 66 (3), p. 193-304.

HASSENZAHL M. (2004). The interplay of beauty, goodness and usability in interactive products. Human Computer Interaction, 19, p. 319-349.

HÛ O., TRIGANO P. (1998). Proposition de critères d'aide à l'évaluation de l'interface homme-machine des logiciels multimédia pédagogiques, $I H M^{\prime} 98$, p 35-42, Nantes.

KIROUAC G. (2004). Cognition et Emotions, Coimbra, Imprensa da Universidade, Portugal.

KIROUAC G. (2004). Cognition et Emotions des systèmes conseillers : une approche méthodologique fondée sur l'analyse du modèle de la tâche, Revue Sciences et Techniques Éducatives, Hermès, Vol. 9 n³-4, p. 157-184.

MATAIGNE B., MAURICE P.-Y. et al. (1995). Guide d'évaluation de logiciels éducatifs. Montréal, Ministère de l'éducation Direction générale des services éducatifs.

www.uqtr.ca/ lamyd/partage/grilles.htm (consulté le 30 avril 2008).

MICHEL G. (2004). Didacticiels accessibles aux personnes présentant une déficience intellectuelle : quelques recommandations. 
Congrès AIRHM, Rimouski, p 128-136, août 2004.

MICHEL G. (2006). Conception et évaluation de didacticiels accessibles aux enfants présentant une déficience intellectuelle in Déficience intellectuelle : savoirs et perspectives d'action, In Presses Inter Universitaires, Québec, p. 289-300.

NCDDR : Projet américain de diffusion de la recherche sur le handicap. www.ncddr.org/du/researchexchange/v08n03 18 access.html (consulté le 30 avril 2008).

NORMAN, D.A. (2002). Emotion and Design : Attractive things work better. Interactions Magazine, IX (4), p. 36-42.

SPERANDIO J. Cl, UZAN G. \& OLTRA R. (1999). L'informatique comme barrière d'exclusion ou comme aide technique à l'intégration, Performances humaines et techniques, $n^{\circ}$ hors-série « Situation et Handicap », p. 33-40, juin 1999.

STEPHANIDIS C., et SAVIDIS A. (2001). Universal Access in the Information Society : Methods, Tools, and Interaction Technologies. Journal Universal Access in the Information Society (UAIS), 2001.

TRICOT, A., DÉTIENNE F., BASTIEN J.M.C. (2003). Recherches en psychologie ergonomique : introduction. Psychologie Française, 48 (3), p. 1-8.

W3C Consortium (2005). Web Accessibility Initiative. http://www.w3.org/WAI/ (consulté le 31 janvier 2006).

WAI-NOT (2007) Projet belge de promotion des TIC auprès des enfants. www.wai-not.org (consulté le 30 avril 2008).

Référence de 1'article :

Gabriel MICHEL, Magali JOBERT, La prise en compte des émotions : une solution pour les logiciels éducatifs pour enfants déficients intellectuels, Rubrique de la revue STICEF, Volume 15, 2008, ISSN : 1764-7223, mis en ligne le 22/01/2009, http://sticef.org

(C) Revue Sciences et Technologies de l'Information et de la Communication pour l'Éducation et la Formation, 2008 